\section{S74 ASSESSING THE DIAGNOSTIC ACCURACY OF THE BRITISH THORACIC SOCIETY ALGORITHM FOR INVESTIGATION OF SOLID PULMONARY NODULES}

${ }^{1}$ A Al-Ameri, ${ }^{2} \mathrm{P}$ Malhotra, ${ }^{3} \mathrm{H}$ Thygesen, ${ }^{4} \mathrm{~S}$ Vaidyanathan, ${ }^{4} \mathrm{~S}$ Karthik, ${ }^{4} \mathrm{~A}$ Scarsbrook, ${ }^{4} \mathrm{M}$ Callister. ${ }^{1}$ Pinderfields General Hospital, Wakefield, UK; ${ }^{2}$ Whiston Hospital, Prescot, UK;

${ }^{3}$ Cancer Research UK Leeds Centre, Leeds, UK; ${ }^{4}$ Leeds Teaching Hospitals, Leeds, UK

\subsection{6/thoraxjnl-2015-207770.80}

Background The British Thoracic Society guidelines (2015) on the investigation and management of pulmonary nodules recommend the use of two risk prediction tools to assess the likelihood of malignancy in solid pulmonary nodules (Brock model following initial CT and the model described by Herder et al. following PET-CT). Management strategies are suggested on the basis of these risk assessments. The aim of this study was to assess the performance of this algorithm in patients with solid pulmonary nodules recruited from a UK teaching hospital.

Method Patients with solid pulmonary nodules $(4-30 \mathrm{~mm})$ were retrospectively identified from the lung cancer MDT and a nodule follow-up clinic $(\mathrm{n}=221)$. All patients had a final diagnosis confirmed by histology or radiological stability on 2 -year follow up.

Results The median age was 69 years. The prevalence of malignancy was $37.1 \%$ (29.9\% primary lung cancer, $7.2 \%$ metastatic disease). 25 patients where PET-CT was recommended by the guideline but did not occur were excluded from subsequent analysis.

Ten patients had nodules $<5 \mathrm{~mm}$ and therefore would have been immediately discharged. All these nodules were benign.

CT surveillance was recommended for 106 patients (37 with nodule $<8 \mathrm{~mm}, 45$ with malignant risk of $<10 \%$ following initial CT, and 24 with malignant risk of $<10 \%$ following PETCT). $94 \%$ of these 106 patients had benign disease, 2\% had primary lung cancer and $4 \%$ had metastatic disease.
Surgical/non-surgical treatment was recommended for 58 patients where the malignant risk was $>70 \%$ following PET-CT. $81 \%$ of these patients had primary lung cancer, $10 \%$ had metastatic disease and $9 \%$ were benign.

For nodules with a malignant risk of between 10 and 70\% following PET-CT, the guidelines recommend consideration of biopsy with alternatives of CT surveillance or surgical resection depending on patient preference and fitness. Of the 22 patients with nodules in this range, $36 \%$ were benign, $55 \%$ primary lung cancer and $9 \%$ metastatic disease.

Conclusion The solid nodule algorithm from the BTS guidelines shows good accuracy in discriminating benign from malignant nodules, recommending appropriate management in a high proportion of cases. Further studies should evaluate this and the other management algorithms with prospectively collected data.

\section{S75 A CLINICAL MODEL TO ESTIMATE THE PROBABILITY OF PULMONARY NODULE MALIGNANCY IN A POPULATION OF ONCOLOGY FOLLOW-UP PATIENTS}

${ }^{1}$ A Talwar, ${ }^{2}$ LC Pickup, ${ }^{2} \mathrm{JMY}$ Willaime, ${ }^{2} \mathrm{M}$ Gooding, ${ }^{1} \mathrm{~T}$ Kadir, ${ }^{1} \mathrm{NM}$ Rahman, ${ }^{1} \mathrm{~F}$ Gleeson. ${ }^{1}$ Oxford University Hospitals NHS Trust, Oxford, UK; ${ }^{2}$ Mirada Medical Ltd, Oxford, UK

\subsection{6/thoraxjnl-2015-207770.81}

Introduction The new BTS Pulmonary Nodule Guidelines 2015 recommend the use of composite prediction models to assess the pre-test probability of malignancy in patients presenting with pulmonary nodules (PNs). These models were not developed for use in patients with a history of malignancy within five years of presentation with a $\mathrm{PN}$.

In order to assist in the diagnosis of PNs, CT texture analysis has been proposed as a potential biomarker in tumour characterisation. ${ }^{1}$ Image texture refers to the statistical analysis of spatial intensity variations of the pixels within an image to produce a CT texture score. ${ }^{2}$

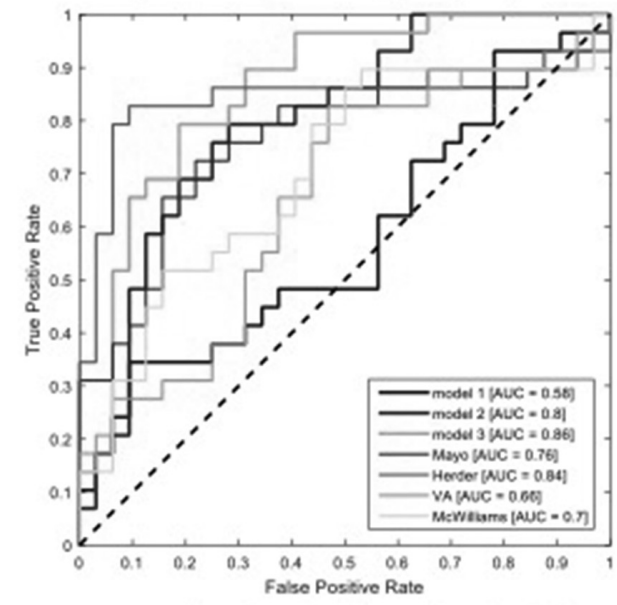

ROC analyses for the different clinical models Model 1: clinical characteristics + diamete Model 2: clinical characteristics + texture score Model 3: texture score

1. Mayo et al 1997. Arch ine Med 157:849-85s 2. Heder et al 2000. Chest 128:2650-2496 3. Goubd et al 2007. Chest 131:383-288

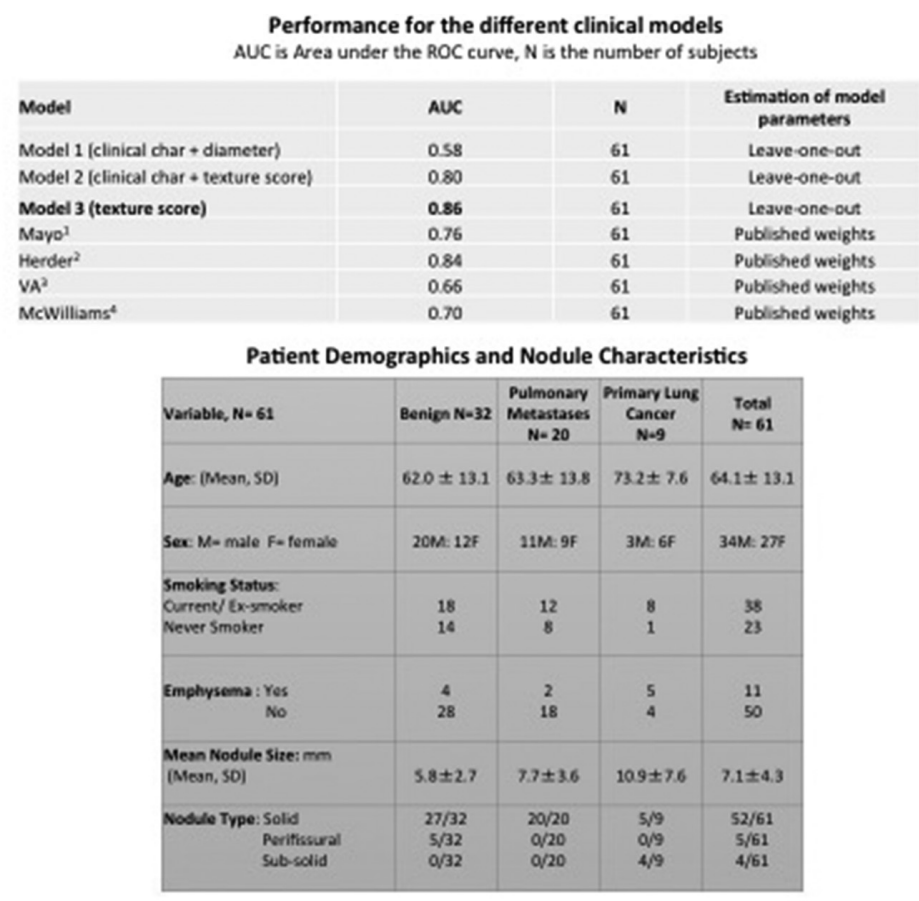

Abstract S75 Figure 1 Comparison of own models to published clinical models for probability of malignancy of pulmonary nodules 